Research Article

\title{
Identification of the Upregulation of MRPL13 as a Novel Prognostic Marker Associated with Overall Survival Time and Immunotherapy Response in Breast Cancer
}

\author{
Hongshan $\mathrm{Ye}^{1}$ and Ning Zhang $\mathbb{D}^{2}$ \\ ${ }^{1}$ Department of Thyroid and Breast Surgery, The 3rd Affiliated Hospital of Shenzhen University, Shenzhen, \\ Guangdong Province, China \\ ${ }^{2}$ Department of Vascular Surgery, The 3rd Affiliated Hospital of Shenzhen University, Shenzhen, Guangdong Province, China
}

Correspondence should be addressed to Ning Zhang; niengch@163.com

Received 13 September 2021; Accepted 5 November 2021; Published 25 November 2021

Academic Editor: Jianxin Shi

Copyright (c) 2021 Hongshan Ye and Ning Zhang. This is an open access article distributed under the Creative Commons Attribution License, which permits unrestricted use, distribution, and reproduction in any medium, provided the original work is properly cited.

\begin{abstract}
Mitochondrial ribosomal protein (MRPL) genes have been reported to participate in many cellular processes, such as cell proliferation, apoptosis, and cell cycle. Meanwhile, the occurrence rate of breast cancer (BRCA) in China steadily increased. Exploring the prognostic value of MRPL genes in BRCA could provide novel biomarkers for BRCA. In this study, to identify prognosis-related genes in breast cancer, the $P$ value and the hazard ratio (HR) of all genes are analyzed with TCGA database. We revealed higher expression level of CEL, PGK1, WNT3A, USP41, LINC02037, PCMT1, LRP11, MCTS1, TCP1, TMEM31, STK4-AS1, STXBP5, LOC100287036, SLC16A2, MRPL13, DERL1, and TARS was correlated to shorter OS time in BRCA. However, higher expression level of JCHAIN, KLRB1, and TNFRSF14 was correlated to longer OS time in BRCA. The further analysis demonstrated MRPL13 was overexpressed in BRCA. Subtype analysis showed that MRPL13 was overexpressed in luminal, HER2-positive BRCA, and TNBC samples and was highest in TNBC samples. Moreover, we revealed higher expression of MRPL13 was significantly correlated to shorter OS time and higher TMB levels in BRCA. Pan-cancer analysis further revealed the prognostic value of MRPL13 in human cancers. MRPL13 expression was significantly increased in multiple human cancers, such as bladder cancer, colon cancer, liver cancer, and prostate cancer. Pan-cancer TMB and overall survival time showed dysregulation of MRPL13 is significantly related to the OS and TMB levels in various cancers. These results further proved that MRPL13 may be a pan-cancer biomarker for predicting prognosis and the response to immunotherapy.
\end{abstract}

\section{Introduction}

Breast cancer (BRCA) consisted of endocrine-dependent breast cancer and HER2-positive breast cancer along with triple-negative breast cancer (TNBC) in view of its histological features [1-3]. Recently, the occurrence rate of BRCA is still increasing, and it reached the highest among women [4, 5]. Besides, the occurrence rate of BRCA amid young people has also heightened [4]. Currently, the occurrence rate of BRCA in China steadily increased [6]. The lethality rate of BRCA accounts for about $18 \%$ of cancer mortality $[1-3,6$, 7]. Present standard treatments against BRCA are mainly composed of surgery, radiotherapy, chemotherapy, hormone therapy, and so on [7]. Targeting receptor tyrosine kinase (RTK) has become an important direction in breast cancer treatment. HER2-targeted treatment therapy significantly improved HER2 ${ }^{+}$BRCA prognosis [8]. Antiestrogen therapy, as the first targeted therapy of human $\mathrm{BC}$, is a treatment of estrogen receptor-positive $\mathrm{BC}[1,8,9]$. Therefore, it is urgently needed to uncover early detection biomarkers and explore therapeutic strategies for BRCA.

Mitochondria function importantly in regulating eukaryotic cells' life and death, which mediates the conversion of aerobic energy via the oxidative phosphorylation (OXPHOS) system and conceals and controls cell apoptosis's internal pathway [10-13]. The mitochondrial ribosome 
comprised a small $28 \mathrm{~S}$ subunit and a large $39 \mathrm{~S}$ subunit [10, 12]. Therefore, mitotic ribosomes are the key to regulating cell respiration. There are some studies showing the levels of gene-encoded mitotic ribosomal proteins, mitotic ribosomal assembly factors, and mitochondrial translation factors have changed in several carcinomas [14-16]. Some researches revealed that these changes were probably associated with the occurrence and metastasis of tumor [15]. In recent years, some of the MRPs participated in multiple cellular processes, including cell proliferation, in vitro ribosome cycle regulation, and apoptosis of cells, and MRPL20 expression levels were significantly downregulated in androgenindependent prostate cancer $[11,12,15]$. The levels of MRPL37 and mRNA in different lymphoma tissues were also significantly increased. MRPL33 is necessary for mitochondrial function and is associated with the progression of tumor $[11,15,17]$. Nonetheless, it is not completely understood of the role of mitochondrial ribosomes in BRCA.

Here, we adopted biology tools and experimental validation of the expression of 33 MRPL family members and their possible. These data indicate that MRPL13 and MRPL18 are involved in the progress of BRCA. In order to verify this possibility, we used bioinformatics to study their expression in BRCA and its relationship with prognosis. A prognostic nomogram model based on seven prognostic-related factors is well used to predict the survival rate and provides new insights for the diagnosis and treatment of BRCA.

\section{Materials and Methods}

2.1. Survival Analysis. We carried out Kaplan-Meier survival analysis and multivariate Cox regression analysis to evaluate the prognostic value of MRPL genes. According to the median of the expression of each gene, we divided patients into highly expressed group and lowly expressed group. The clinical factors associated with BRCA's prognosis and clinical indicators were separately analyzed by KaplanMeier (KM) survival analysis and Cox regression analysis [18]. The Kaplan-Meier plotter is a database used for analyzing the correlation between genes and survival time in 21 cancer types.

2.2. Data Preparation. We analyzed mRNA expression data from The Cancer Genome Atlas (TCGA, http://tcga-data .nci.nih.gov/tcga) [19].

2.3. Bioinformatics Analysis. To determine the distribution of MRPL genes in BRCA tumors and normal tissues, we conducted the gene expression profile interaction analysis (GEPIA) (http://gepia.cancer-pku.cn/) to obtain a box diagram of MRPL genes [20]. We carried out annotation, visualization and integrated discovery database (DAVID) v.6.8 [21], and BINGO (https://www.psb.ugent.be/cbd/papers/ $\mathrm{BINGO} /$ Home) [22] to analyze enriched functions.

2.4. MRPL-Related Gene Analysis in BRCA. We applied Pearson correlation coefficient analysis [23] to evaluate the MRPL-associated genes in BRCA. The correlation coefficient $R \geq 0.4$ or $R \leq-0.4$ is considered highly correlated. $P$ value $\leq$ 0.01 means significantly statistical difference.
2.5. Clinical Significance of $M R P L$ Genes in BRCA. In view of the median of the expression of each gene, we divided patients into highly expressed group and lowly expressed group. The Kaplan-Meier estimator is used to determine the correlation of gene expression with patients' overall survival rate (OS). We adjusted the multivariate Cox proportional hazards regression model in the light of tumor stage.

2.6. Nomogram for Predicting BRCA's Prognosis. We constructed nomogram to predict BRCA's prognosis and risk scores. This model contained all MRPL genes and clinical details. We calculated the points corresponding to each parameter and then evaluated the correlation of obtained points with the risk. We predicted 1-year, 5-year, and 10year prognostic status [24, 25].

2.7. Statistical Analysis. We evaluated the differences in survival rates existing in the two groups according to hazard ratios (HRs) and 95\% confidence intervals (CIs). SPASS version 25.0 (IBM, USA) and Graphpad version 7.0 (LaJolla, USA) were applied for statistical analysis and mapping. $P$ $<0.05$ means greatly statistical differences between the two groups.

\section{Results}

3.1. Identification of Prognosis-Related Genes in Breast Cancer. In order to identify prognosis-related genes in breast cancer, the $P$ value and the hazard ratio (HR) of all genes are analyzed with TCGA database. The top 20 significantly genes related to the prognosis of breast cancer are listed in Figure 1. Among them, higher expression level of CEL, PGK1, WNT3A, USP41, LINC02037, PCMT1, LRP11, MCTS1, TCP1, TMEM31, STK4-AS1, STXBP5, LOC100287036, SLC16A2, MRPL13, DERL1, and TARS was correlated to shorter OS time in BRCA (Figure 1). However, higher expression level of JCHAIN, KLRB1, and TNFRSF14 was correlated to longer OS time in BRCA (Figure 1). Interestingly, we observed several lncRNAs were significantly related to prognosis in breast cancer, such as LINC02037 and STK4-AS1. Among these genes, we focused on MRPL13, whose function in BRCA remained largely unclear.

3.2. The Expression and Protein Levels of MRPL13 Were Upregulated in BRCA. In order to further confirm the upregulation of MRPL13 in BRCA, we used the UALCAN database to evaluate MRPL13 gene and protein levels in BRCA. Figure 2 illustrates that MRPL13 RNA and protein levels in BRCA are significantly higher than normal samples (Figures 3(a) and 3(b)). Additionally, subtype analysis showed that MRPL13 was overexpressed in luminal, HER2-positive BRCA, and TNBC samples and was higher in TNBC samples than the two former samples (Figures 3(c) and 3(d)).

3.3. Constructing and Evaluating the Nomogram for MRPL13. We also analyzed univariate and multivariate Cox proportional hazards and found that three general variables (age, gender, and TNM staging) and two genes 


\begin{tabular}{lrcr} 
Names & p.value & Hazard ratio $(95 \% \mathrm{CI})$ \\
\hline CEL & $<0.0001$ & $1.358(1.222,1.509)$ \\
PGK1 & $<0.0001$ & $1.798(1.439,2.247)$ \\
WNT3A & $<0.0001$ & $1.707(1.354,2.152)$ \\
USP41 & $<0.0001$ & $1.529(1.267,1.845)$ \\
LINC02037 & $<0.0001$ & $1.896(1.422,2.528)$ \\
PCMT1 & $<0.0001$ & $1.806(1.379,2.365)$ \\
LRP11 & $<0.0001$ & $1.569(1.276,1.93)$ \\
MCTS1 & $<0.0001$ & $2.17(1.516,3.106)$ \\
TCP1 & $<0.0001$ & $1.652(1.304,2.094)$ \\
TMEM31 & $<0.0001$ & $1.579(1.268,1.966)$ \\
JCHAIN & $<0.0001$ & $0.886(0.835,0.939)$ \\
KLRB1 & $<0.0001$ & $0.733(0.629,0.854)$ \\
STK4-AS1 & $<0.0001$ & $1.969(1.405,2.759)$ \\
STXBP5 & $<0.0001$ & $1.575(1.249,1.985)$ \\
LOC100287036 & $<0.0001$ & $1.959(1.389,2.764)$ \\
SLC16A2 & $<0.0001$ & $1.33(1.15,1.54)$ \\
TNFRSF14 & $<0.0001$ & $0.698(0.58,0.839)$ \\
MRPL13 & $<0.0001$ & $1.507(1.22,1.863)$ \\
DERL1 & $<0.0001$ & $1.561(1.237,1.971)$ \\
TARS & $<0.0001$ & $1.58(1.243,2.007)$ \\
& &
\end{tabular}

FIgURE 1: Identification of prognosis-related genes in breast cancer. The top 20 significantly genes related to the prognosis of breast cancer are listed in Figure 1.

(MRPL13 and MRPL18) presented great difference existing in the nonrelapsed and the relapsed groups (Figures 2(a) and $2(\mathrm{~b}))$. We constructed a nomogram to calculate the recurrence risk of each patient through the points related to MRPL13 and MRPL18 on the basis of the multivariate Cox proportional hazard analysis. The total score is $0-160$ points, and the variables are calculated and combined (Figure 2(c)). After comparison with the expected model in the whole cohorts, we concluded that the calibration plots for 1-year, 3-year, and 5-year OS rates presented well prediction (Figure 2(d)).

3.4. Overexpression of MRPL13 Was Correlated to Shorter OS in BRCA. Next, we conducted further analysis on MRPL13 in BRCA. According to the median expression of MRPL13 in BRCA, we separated BRCA patients into MRPL13 highly expressed group and MRPL13 lowly expressed group. Figure 4(a) shows that high expression levels are associated with more deaths. KM survival curves showed that BRCA patients with highly expressed MRPL13 exhibited shorter OS than those with lowly expressed MRPL13 (Figure 4(b)). The AUC values of 1-year, 3-year, 5-year, 10-year, and 15year survival rate prediction features are 0.605, 0.605, $0.595,0.586$, and 0.528 , respectively (Figure $4(\mathrm{c})$ ).

3.5. The Confirmation of Correlation between MRPL13 Expression and Prognosis. Next, we use the KM database to confirm the association of MRPL13 expression with prognosis. Our results show that BRCA patients with highly expressed MRPL13 displayed shorter OS, RFS, PPS, and DMF time (Figures 5(a)-5(d)). Further, subtype analysis showed that luminal A, luminal B, of HER2-positive, and TNBC patients with high levels of MRPL13 expression had shorter RFS time (Figures 5(e)-5(h)).

3.6. Overexpression of MRPL13 Was Correlated to Higher $T M B$ Levels in BRCA. Tumor mutational burden (TMB) is a promising biomarker for predicting tumor immunotherapy response. Here, we assessed the relationship of MRPL13 expression with TMB levels. Our results show that higher expression of MRPL13 was correlated to higher TMB levels in BRCA, luminal A BRCA, luminal B BRCA, and HER2enriched BRCA patients (Figures 6(a)-6(d)). Nevertheless, there is no greatly significant correlation of MRPL13 with TMB level in TNBC patients (Figure 6(e)).

3.7. Expression Levels of MRPL13 Were Correlated to OS Time and TMB Levels in Pan-Cancer. In addition, three different databases based on TCGA cohort were used to evaluate the expression of MRPL13 mRNA in 33 cancer types. TIMER database results have shown that MRPL13 expression in certain cancers were significantly increased, including the BLCA, the BRCA, CESC, CHOL, COAD, ESCA, GBM, HNSCs, LIHC, LUAD, LUSC, PRAD, Read, the STAD, and UCEC (Figures $7(\mathrm{a})-7(\mathrm{~d})$ ). However, MRPL13 expression was reduced in $\mathrm{KICH}, \mathrm{KIRC}$, and PCPG (Figures $7(\mathrm{a})-7(\mathrm{~d}))$.

We also analyzed the association of MRPL13 expression in pan-carcinoma MRPL13 expression with TMB and OS. Our results show that the dysregulation of MRPL13 is significantly related to the OS of ACC, BRCA, KICH, LAML, LGG, LIHC, LUAD, PAAD, SARC, and UVM (Figure 8(a)). 


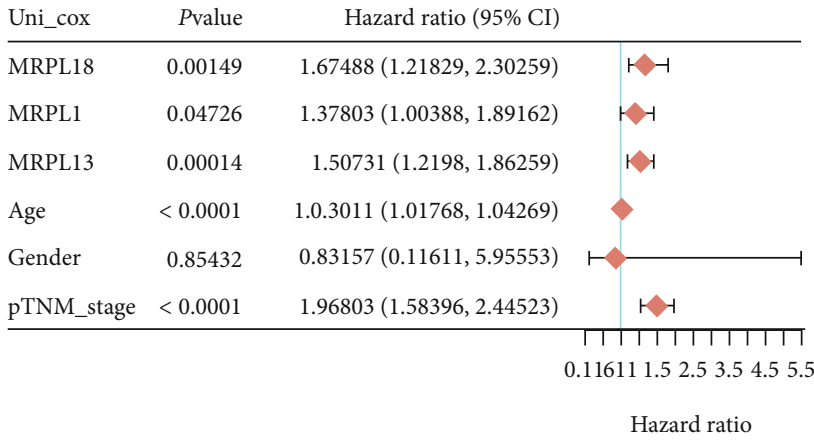

(a)

Points

MRPL18

MRPL13

Total ponts

Linear predictor
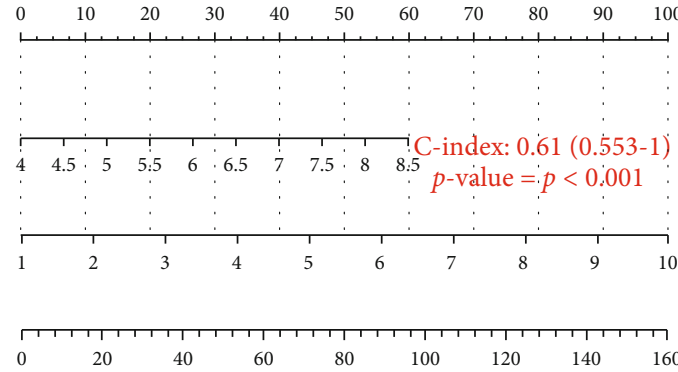

1-year survival pro

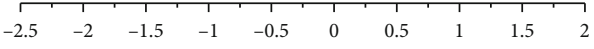

3-year survival pro

5-year survival pro

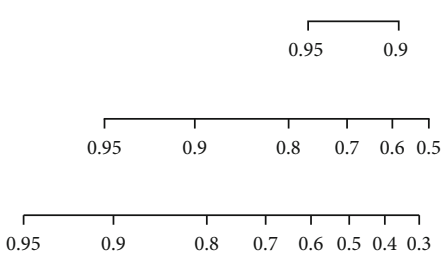

(c)

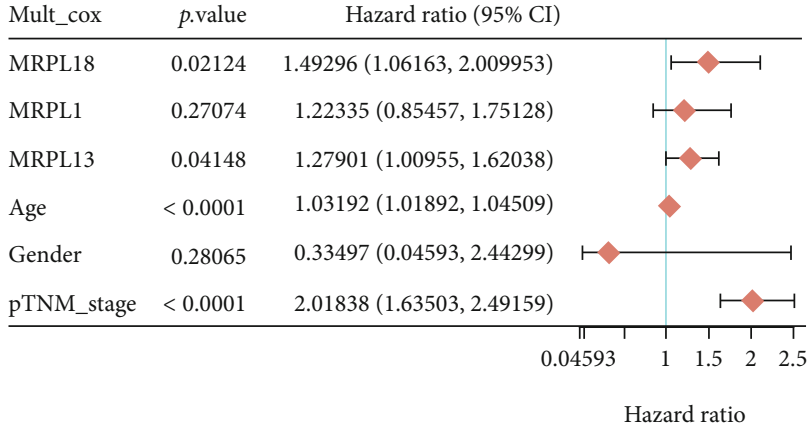

(b)

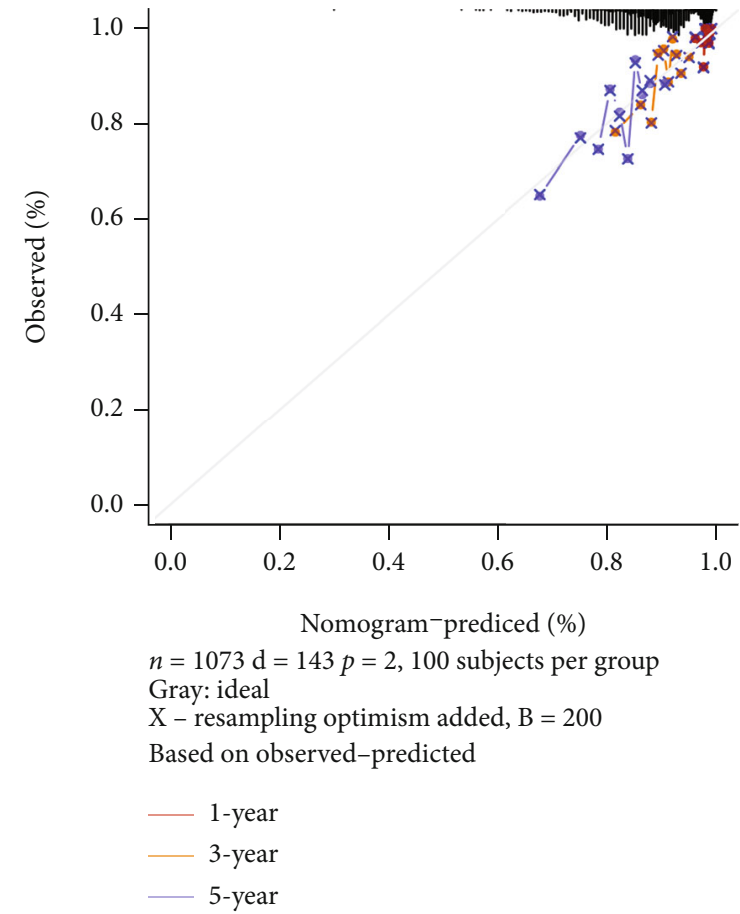

(d)

FIGURE 2: Constructing and evaluating the nomogram for MRPL13. (a) Univariate analysis and (b) multivariate analysis of the correlation between MRPL13 and overall survival in BRCA. (c) The nomogram is constructed for MRPL13 in BRCA. (d) Calibration curves of the nomogram for the prediction of survival rates at 1,3 , and 5 years.

In addition, we also analyzed the correlation of MRPL13 expression with TMB in human cancer. Our results show that MRPL13 expression exhibited a significantly positive correlation with TMB levels of DLBC, STAD, PAAD, BRCA, LUAD, UCS, SARC, LGG, PRAD, and LUSC, while presented a significantly negative correlation with the TMB levels of thymus, UVM. These results further prove that MRPL13 may be a pan-cancer biomarker for predicting response to immunotherapy (Figure 8(b)).

\section{Discussion}

In this study, we identified prognosis-related genes in breast cancer. Interestingly, we observed several lncRNAs were significantly related to prognosis in breast cancer, such as LINC02037 and STK4-AS1. Previous studies had revealed these lncRNAs have a crucial role in human cancers. For example, Li et al. reported LINC02037 was differently expressed in breast cancer and could predict prognosis of breast cancer patients. In recent years, some MRPs have been reported to participate in many cellular processes, such as cell proliferation, apoptosis, and cell cycle [10, 14-16]. The growing researches have reported that the abnormally expressed MRPs exhibited a relationship to human tumors' occurrence and development $[15,16]$. For example, MRPL15 is a new prognostic indicator and therapeutic target for epithelial ovarian cancer [26]. MRPS12 is a potential oncogene of ovarian cancer [27], while MRPL27 displays an adverse effect on the overall survival rate and disease-free survival rate of cholangiocarcinoma patients [28]. MRPL42 is activated by YY1 to promote the progression of lung adenocarcinoma [29]. MRPL42 gene knockout inhibits the proliferation of glioma cells by inducing cell cycle arrest and apoptosis 


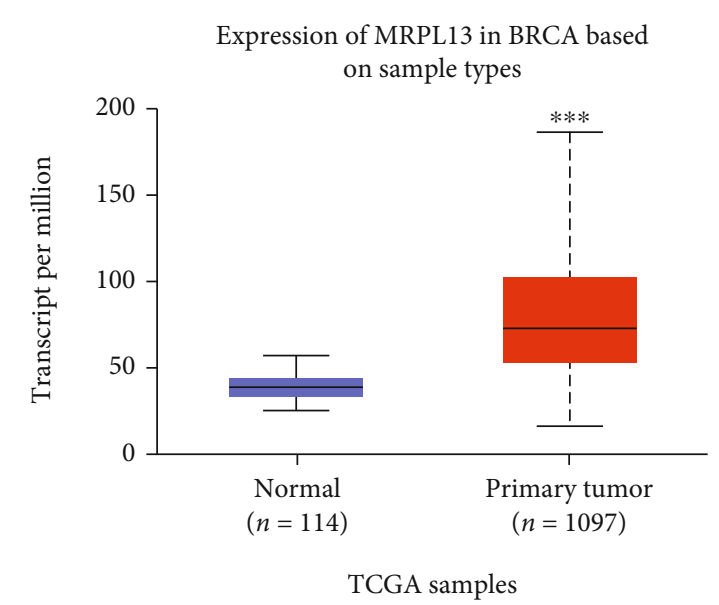

(a)

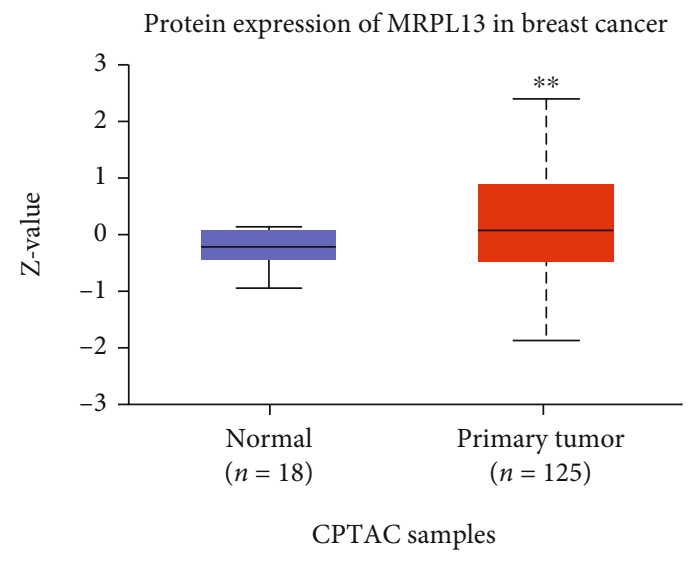

(c)

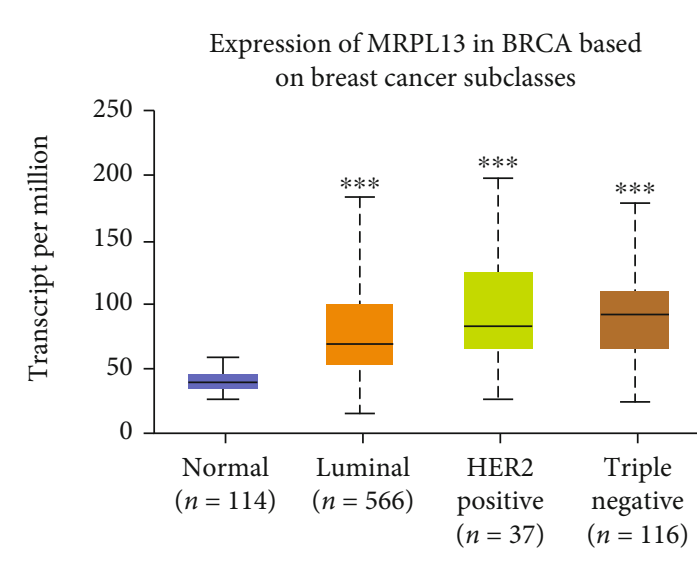

TCGA samples

(b)

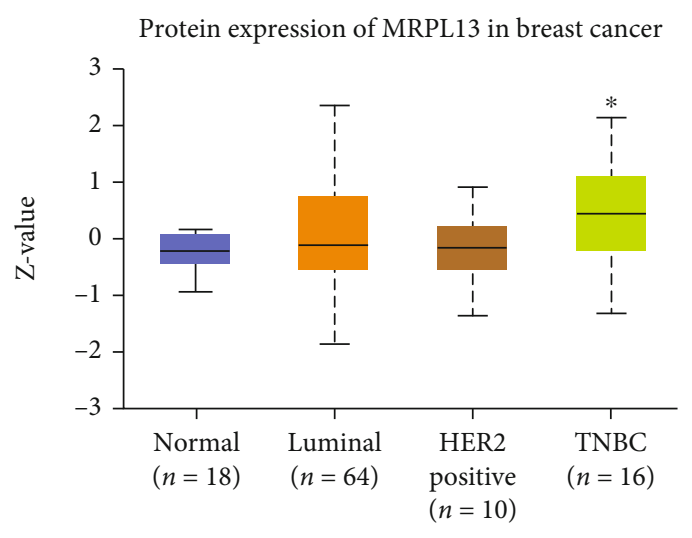

CPTAC samples

(d)

Figure 3: MRPL13 is overexpressed in BRCA. (a) The RNA levels of MRPL13 are overexpressed in BRCA compared to normal samples. (b) The RNA levels of MRPL13 are different among normal samples, luminal BRCA, HER2-positive BRCA, and TNBC. (c) The protein levels of MRPL13 are overexpressed in BRCA compared to normal samples. (d) The protein levels of MRPL13 are different among normal samples, luminal BRCA, HER2-positive BRCA, and TNBC. The $P$ value was calculated by the unpaired two-tailed Student's $t$-test. ${ }^{*} P<0.05$, ${ }^{* *} P$ $<0.01$, and ${ }^{* * *} P<0.001$.

[30]. Here, we analyzed TCGA data and performed bioinformatics to reveal potential prognostic value of MRPL genes in BRCA. The results indicate significant difference exists in MRPL family gene (including MRPL1, MRPL13, and MRPL18) expression between BRCA tissues and normal tissues. Survival analysis showed that the overexpression of MRPL1, MRPL13, and MRPL18 was associated with a shorter OS time in BRCA.

Mitochondrial ribosomal protein L13 (MRPL13) is located on chromosome 6 and encodes the 39 S large subunit of mitochondrial ribosomes [31,32]. MRPL13 is a predictive biomarker of BRCA and exhibits a relationship to the immune infiltration of BRCA. MRPL13 mediated the promotion of BRCA cells' proliferation, migration, and EMT process through PI3K-AKT-mTOR pathway [32]. siRNAmediated knockout of MRPL13 reduces the expression of mitochondrial protein in SNU387 cells, reduces oxygen consumption, and increases CLN1-mediated tumor cell inva- siveness [33-35]. Nevertheless, it is still elusive towards MRPL13's role in BRCA. Here, we tested MRPL13 expression in normal tissues, various cell lines, and pan-cancer. Our results show that the expression of MRPL13 in BRCA is higher than that in normal people. Subtype analysis shows that MRPL13 is overexpressed in luminal, HER2-positive, and TNBC samples and is higher in TNBC samples than the former samples. Moreover, high expression of MRPL13 is related to poor prognosis of BRCA. These results suggest MRPL13 is a potential marker for BRCA prognosis.

Traditionally, BRCA is considered as a low immunogenic disease (so-called "cold" tumor) because of its low mutation burden, low number of tumor infiltrating lymphocytes (TIL), and low expression of programmed cell death protein/ligand (PD-1/L1) [36-38]. Preclinical and clinical trials have shown that immunotherapy is an emerging method for BRCA treatment. Recently, nivolumab (anti$\mathrm{PD}-1 \mathrm{mAb}$ ) and ipilimumab (anti-CTLA-4-mAb) have been 

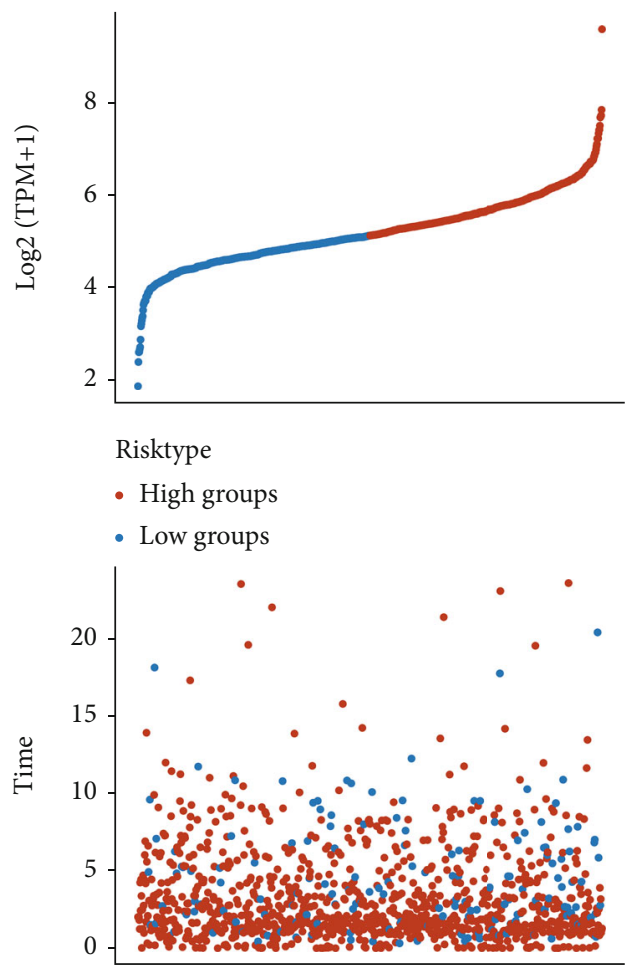

Status

- Alive

- Dead

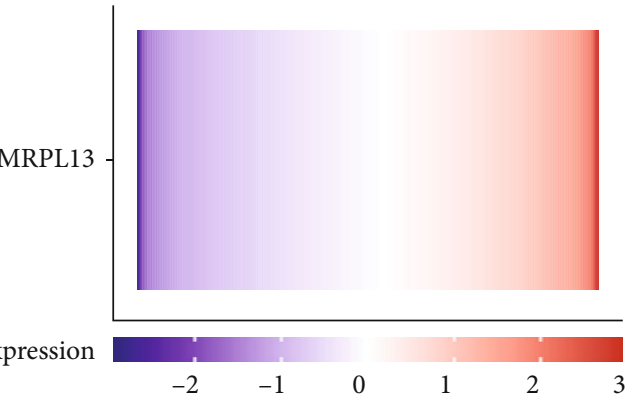

(a)

Figure 4: Continued. 


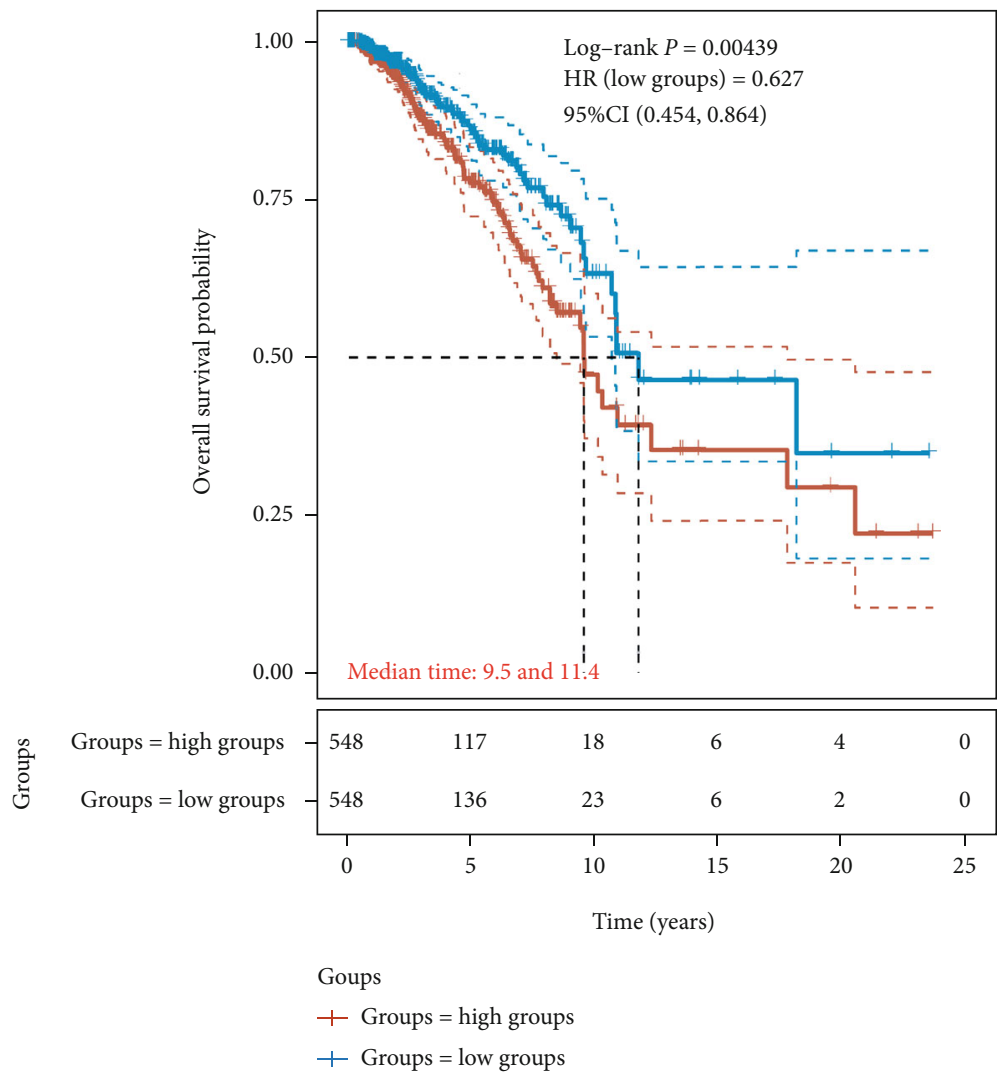

(b)

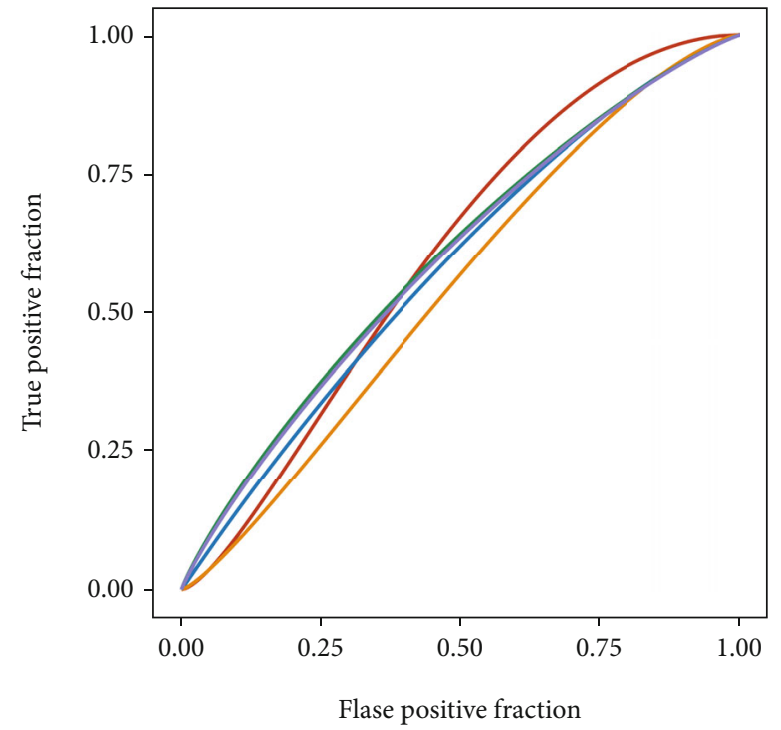

Type

- 1-years, AUC $=0.605,95 \% \mathrm{CI}(0.515-0.694)$

- 10-years, AUC $=0.586,95 \% \mathrm{CI}(0.495-0.676)$

— 15-years, AUC $=0.528,95 \% \mathrm{CI}(0.366-0.69)$

- 3-years, AUC $=0.605,95 \% \mathrm{CI}(0.543-0.667)$

— 5-years, AUC $=0.595,95 \% \mathrm{CI}(0.537-0.653)$

(c)

FIgURE 4: Higher expression level of MRPL13 was correlated to shorter OS in BRCA. (a) From top to bottom are the expression values of MRPL13, patients' survival status distribution, and the heatmap of MRPL13 expression in the low and high groups. (b) Higher expression level of MRPL13 was correlated to shorter OS in BRCA. (c) AUC analysis of MRPL13 in BRCA. 


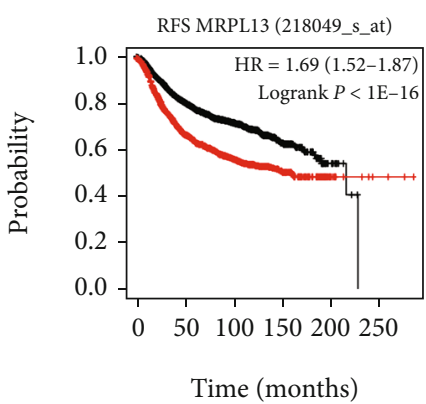

(a)

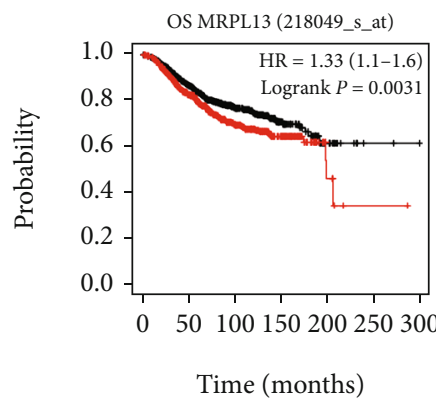

(c)

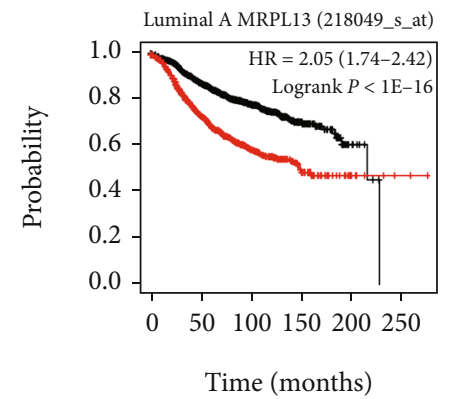

(e)

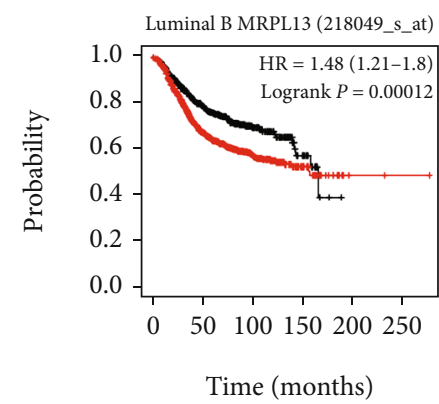

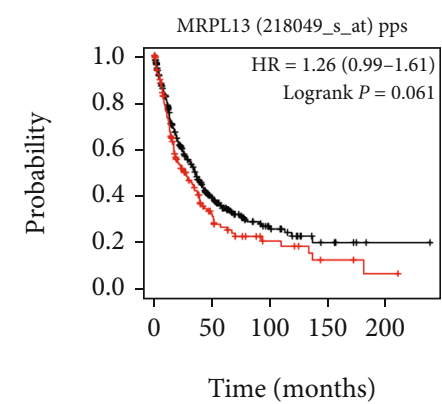

(b)

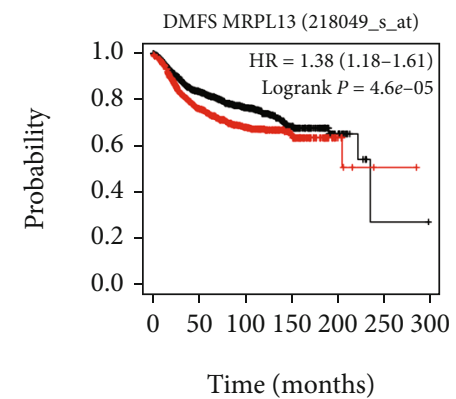

(d)

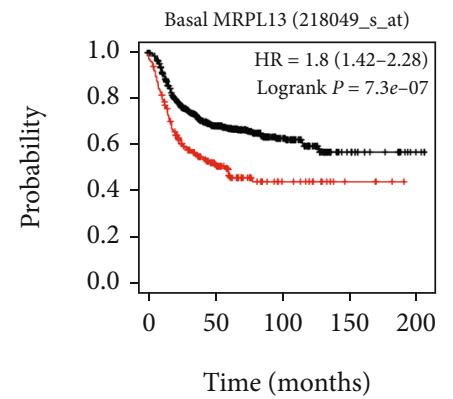

(f)

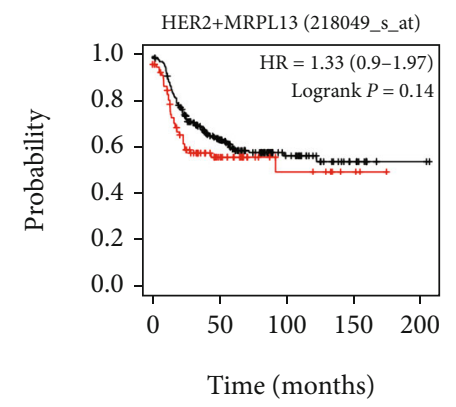

Expression

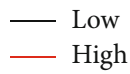

(h)

FIGURE 5: The confirmation of correlation between MRPL13 expression and prognosis. (a-d) highly expressed MRPL13 displayed shorter (a) RFS, (b) PPS, (c) OS, and (d) DMFS time. (e-h) Subtype analysis showed that (e) luminal A, (g) luminal B, (h) HER2-positive, and (f) TNBC patients with high levels of MRPL13 expression had shorter RFS time.

applied in phase I/II trial of TNBC treatment and achieved well clinical efficacy [36-40]. The use of several immunotherapy agents as a single treatment for metastatic triplenegative BRCA has also shown a moderate but long-lasting response rate and tolerable safety $[40,41]$. However, the clinical application of immunotherapy is limited because the efficacy of immunotherapy agents is still very low [41, 42]. Therefore, there is an urgent need to use new 


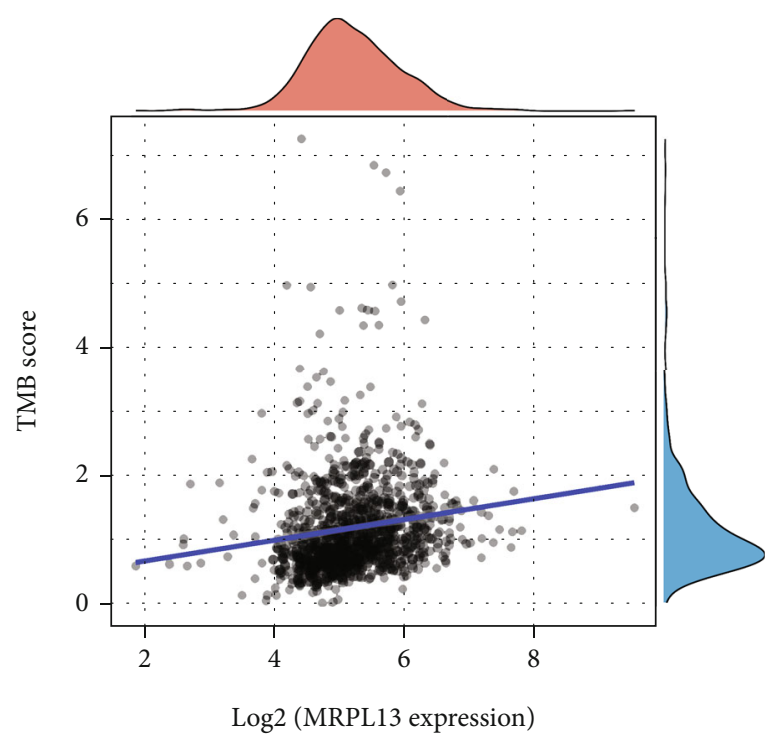

(a)

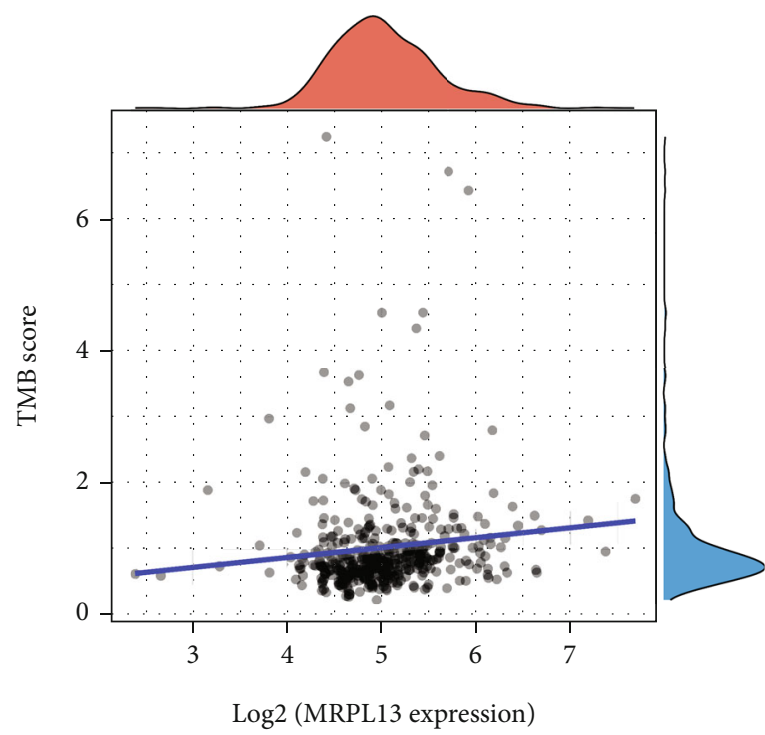

(c)

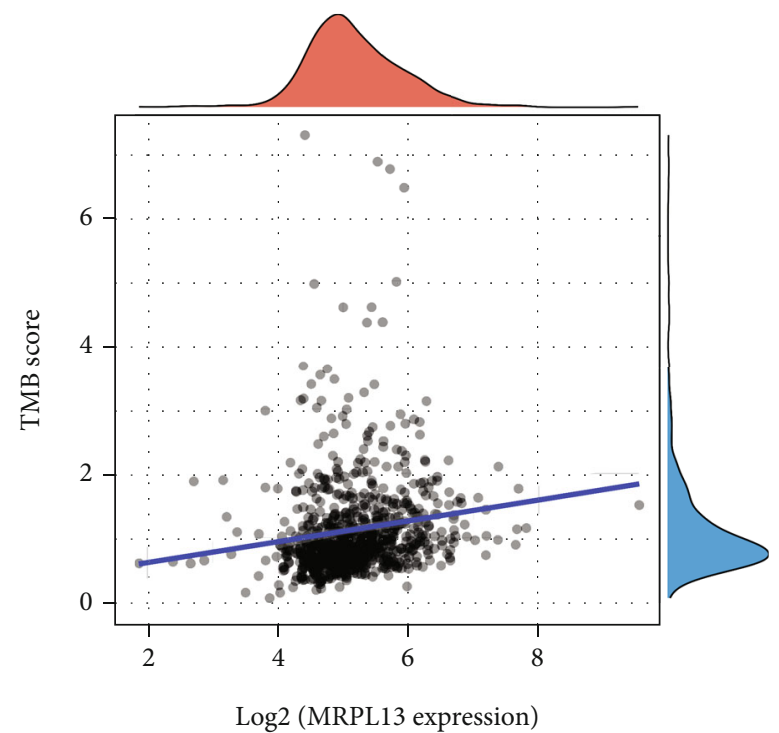

(b)

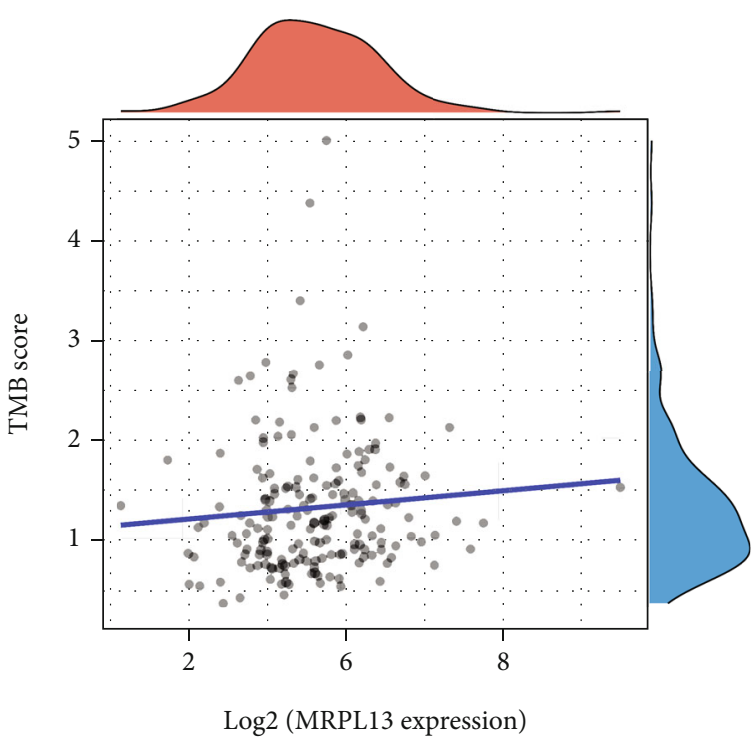

(d)

FIgURe 6: Continued. 


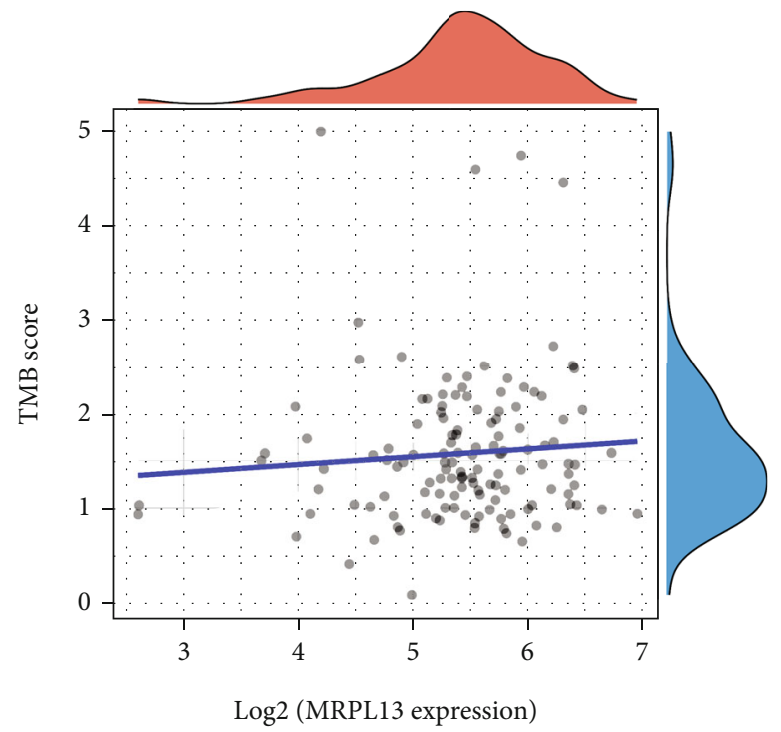

(e)

FiguRE 6: Higher expression of MRPL13 was correlated to higher TMB levels in BRCA. (a-e) Our results show expression of MRPL13 was correlated to TMB levels in BRCA, luminal A BRCA, luminal B BRCA, HER2-enriched BRCA, and TNBC patients.

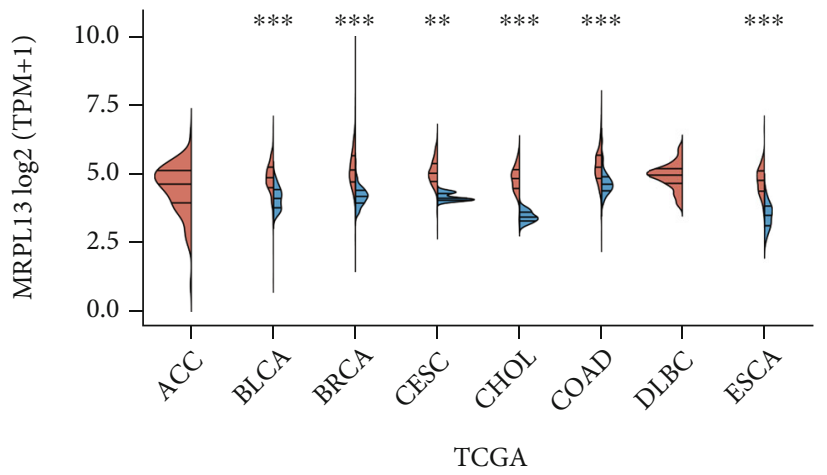

(a)

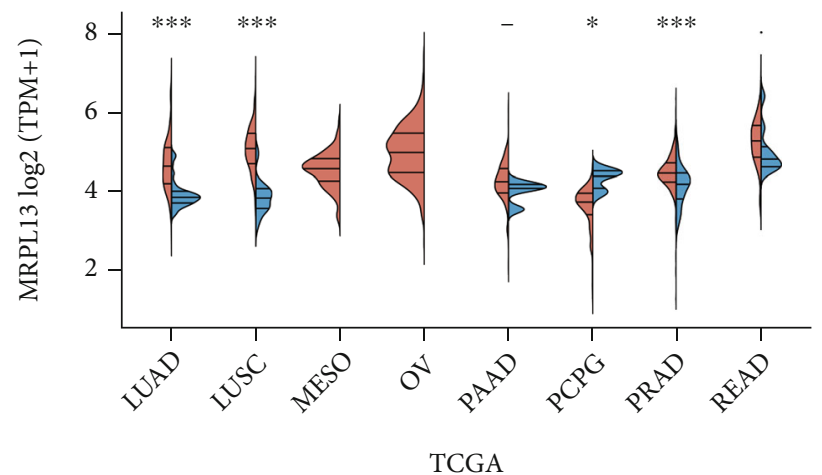

(c)

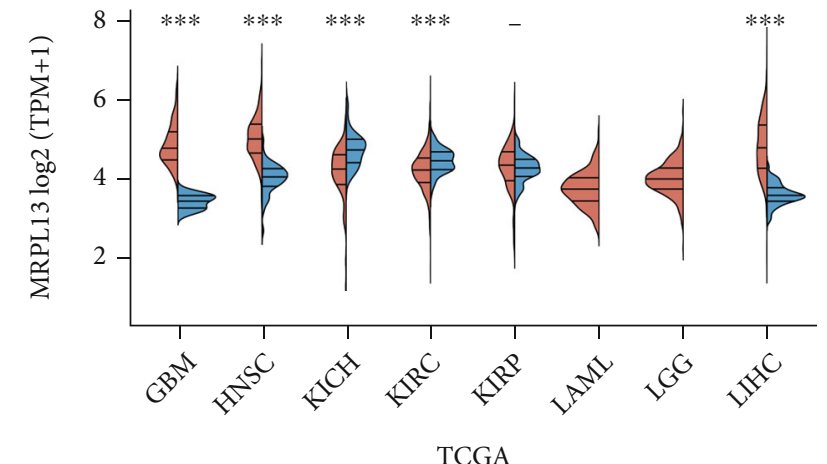

(b)

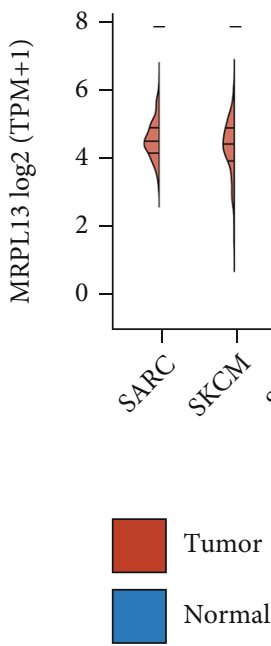

Normal

TCGA

FIGURE 7: Pan-cancer analysis showed MRPL13 was upregulated in BRCA. 


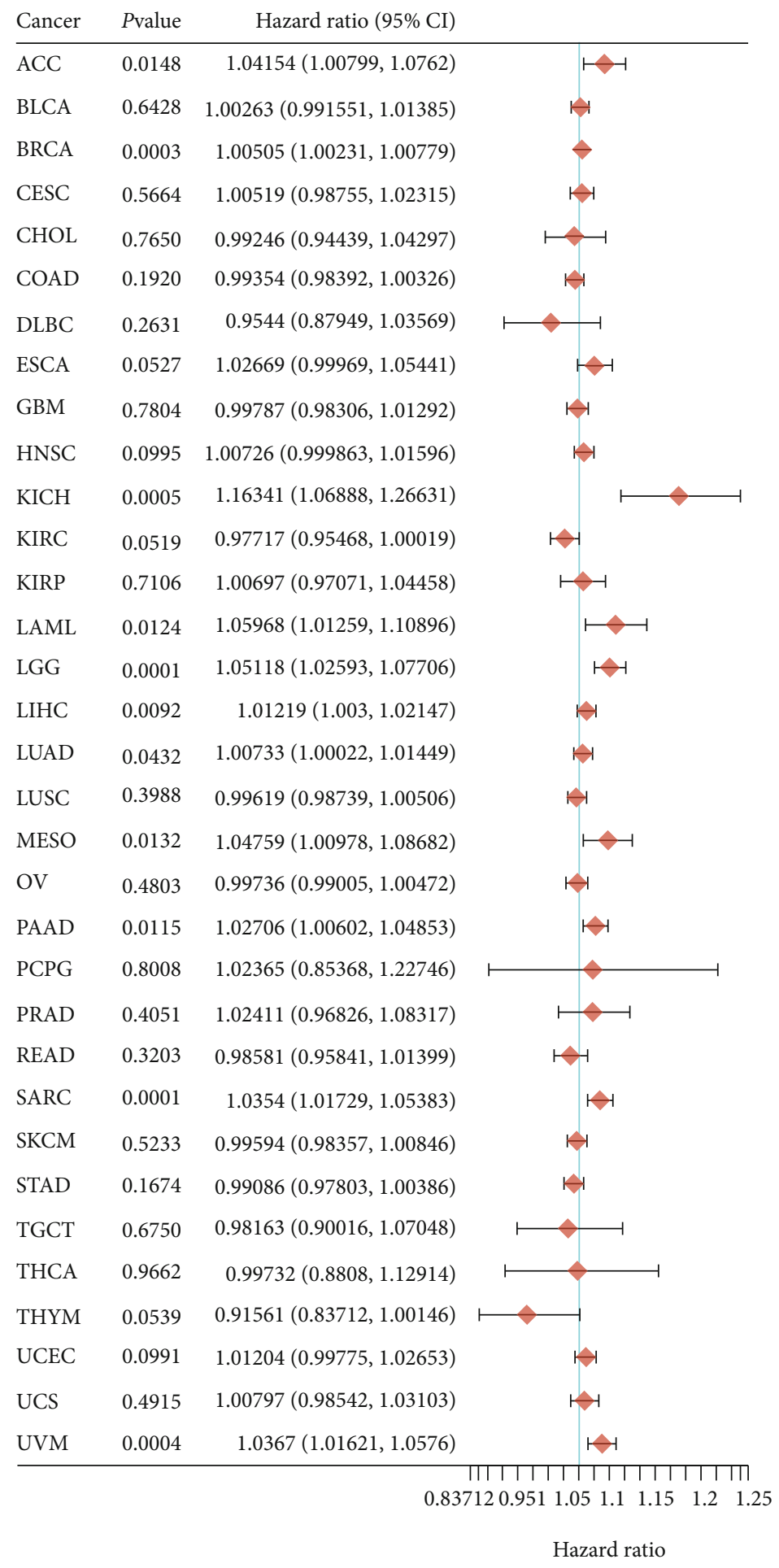

(a)

FIGURE 8: Continued. 

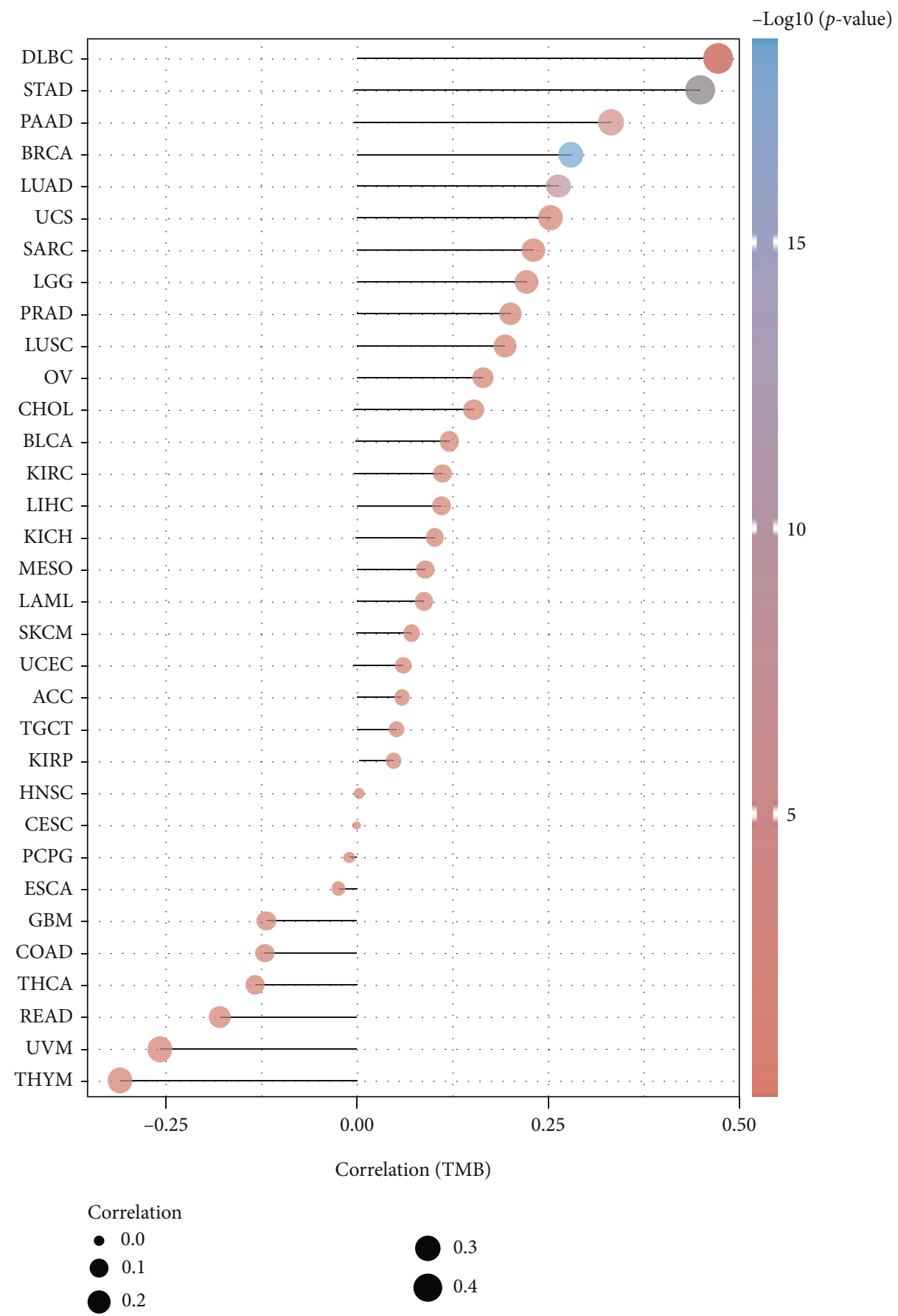

(b)

FIgURE 8: Pan-cancer analysis showed MRPL13 was correlated to OS and TMB levels in human cancers. (a) Pan-cancer analysis showed MRPL13 was correlated to OS in human cancers. (b) Pan-cancer analysis showed MRPL13 was correlated to TMB levels in human cancers.

biomarkers to predict the response of BRCA to immunotherapy. TMB is related to a variety of tumor immunogenicity indicators and has been proven to have clinical application value in carcinomas [43]. Higher TMB value was observed in TNBC than other subtypes of BRCA. Recently, some studies show that TNBC cases with high TMB perhaps benefit from immune checkpoint blockade along with chemotherapy or immune checkpoint blockade alone [40-44]. As a potential toolset, TMB could be utilized as a helper for TNBC patients [44]. Currently, we firstly demonstrated that increase level of MRPL13 is significantly related to the increase of TMB value in TNBC. In addition, we also analyzed MRPL13 with TMB relation human cancers, and the results show that MRPL13 and DLBC, STAD, PAAD, BRCA, LUAD, UCS, SARC, LGG, PRAD, and LUSC in $\mathrm{TMB}$ were significantly positively correlated with elevated levels. And it is negatively correlated with the increase of TMB levels in thymus, UVM. These results further prove 
that MRPL13 may be a pan-cancer biomarker for predicting response to immunotherapy.

Finally, this study has some limitations. First of all, this research is based on bioinformatics analysis, and its potential biological mechanism needs to be further studied. Second, we would conduct more researches to investigate the protein expression level of MRPL13 and its role in the pathogenesis and progression of BRCA. We firstly revealed the potential association of MRPL gene expression with BRCA tumor immune escape and fully explored the role of MRPL gene in BRCA patients. Our findings would offer some certain reference value for the research and clinical treatment of BRCA.

In short, the high expression of MRPL3 predicts a poor prognosis of BRCA, so it may be a potential biomarker of the disease. These genes can regulate BRCA tumor-related signaling pathways and inhibit the immune infiltration of BRCA tumors. Further prospective studies are needed to verify these molecular mechanisms.

\section{Data Availability}

The data used in the current study are available from the GEO (https://www.ncbi.nlm.nih.gov) and TCGA (https:// portal.gdc.cancer.gov/).

\section{Conflicts of Interest}

The authors declare that there are no competing interests.

\section{References}

[1] J. A. Fernandez-Pol, P. D. Hamilton, and D. J. Klos, "Genomics, proteomics and cancer: specific ribosomal, mitochondrial, and tumor reactive proteins can be used as biomarkers for early detection of breast cancer in serum," Cancer Genomics \& Proteomics, vol. 2, no. 1, pp. 1-24, 2005.

[2] G. N. Hortobagyi and A. U. Buzdar, "Current status of adjuvant systemic therapy for primary breast cancer: progress and controversy," CA: A Cancer Journal for Clinicians, vol. 45, no. 4, pp. 199-226, 1995.

[3] C. R. Smart, C. Byrne, R. A. Smith et al., "Twenty-year followup of breast cancers diagnosed during breast cancer demonstration project," CA: A Cancer Journal for Clinicians, vol. 47, no. 3, pp. 134-149, 1997.

[4] M. F. Ullah, "Breast cancer: current perspectives on the disease status," Advances in Experimental Medicine and Biology, vol. 1152, pp. 51-64, 2019.

[5] W. D. Foulkes, I. E. Smith, and J. S. Reis-Filho, "Triple-negative breast cancer," The New England Journal of Medicine, vol. 363, no. 20, pp. 1938-1948, 2010.

[6] K. Yang, Y. Sheng, C. Huang et al., "Clinical characteristics, outcomes, and risk factors for mortality in patients with cancer and COVID-19 in Hubei, China: a multicentre, retrospective, cohort study," The Lancet Oncology, vol. 21, no. 7, pp. 904913, 2020.

[7] L. Rossi, C. Mazzara, and O. Pagani, "Diagnosis and treatment of breast cancer in young women," Current Treatment Options in Oncology, vol. 20, no. 12, p. 86, 2019.
[8] G. Anandappa and N. C. Turner, "Targeting receptor tyrosine kinases in HER2-negative breast cancer," Current Opinion in Oncology, vol. 25, no. 6, pp. 594-601, 2013.

[9] M. Szostakowska, A. Trębińska-Stryjewska, E. A. Grzybowska, and A. Fabisiewicz, "Resistance to endocrine therapy in breast cancer: molecular mechanisms and future goals," Breast Cancer Research and Treatment, vol. 173, no. 3, pp. 489-497, 2019.

[10] E. Klæstad, S. Opdahl, M. J. Engstrøm et al., "MRPS23 amplification and gene expression in breast cancer; association with proliferation and the non-basal subtypes," Breast Cancer Research and Treatment, vol. 180, no. 1, pp. 73-86, 2020.

[11] M. L. Gatza, G. O. Silva, J. S. Parker, C. Fan, and C. M. Perou, "An integrated genomics approach identifies drivers of proliferation in luminal-subtype human breast cancer," Nature Genetics, vol. 46, no. 10, pp. 1051-1059, 2014.

[12] N. Kenmochi, T. Suzuki, T. Uechi et al., "The human mitochondrial ribosomal protein genes: mapping of 54 genes to the chromosomes and implications for human disorders," Genomics, vol. 77, no. 1-2, pp. 65-70, 2001.

[13] H.-J. Kim, P. Maiti, and A. Barrientos, "Mitochondrial ribosomes in cancer," Seminars in Cancer Biology, vol. 47, pp. 67-81, 2017.

[14] V. Gogvadze, S. Orrenius, and B. Zhivotovsky, "Mitochondria in cancer cells: what is so special about them?," Trends in Cell Biology, vol. 18, no. 4, pp. 165-173, 2008.

[15] D. C. Wallace, "Mitochondria and cancer," Nature Reviews. Cancer, vol. 12, no. 10, pp. 685-698, 2012.

[16] O. Warburg, "On the origin of cancer cells," Science, vol. 123, no. 3191, pp. 309-314, 1956.

[17] J. Li, D. Feng, C. Gao et al., "Isoforms S and L of MRPL33 from alternative splicing have isoform-specific roles in the chemoresponse to epirubicin in gastric cancer cells via the PI3K/AKT signaling pathway," International Journal of Oncology, vol. 54, no. 5, pp. 1591-1600, 2019.

[18] S. Poornakala and N. S. Prema, "A study of morphological prognostic factors in colorectal cancer and survival analysis," Indian Journal of Pathology \& Microbiology, vol. 62, no. 1, pp. 36-42, 2019.

[19] W. Zhao, D. Geng, S. Li, Z. Chen, and M. Sun, "LncRNA HOTAIR influences cell growth, migration, invasion, and apoptosis via the miR-20a-5p/HMGA2 axis in breast cancer," Cancer Medicine, vol. 7, no. 3, pp. 842-855, 2018.

[20] T. Zheng, A. Wang, D. Hu, and Y. Wang, "Molecular mechanisms of breast cancer metastasis by gene expression profile analysis," Molecular Medicine Reports, vol. 16, no. 4, pp. 4671-4677, 2017.

[21] J. Chen, C. Liu, J. Cen et al., "KEGG-expressed genes and pathways in triple negative breast cancer," Medicine, vol. 99, no. 18, 2020.

[22] C. Gao, H. Li, J. Zhuang et al., "The construction and analysis of ceRNA networks in invasive breast cancer: a study based on The Cancer Genome Atlas," Cancer Management and Research, vol. 11, pp. 1-11, 2019.

[23] Y. Ma, Y. Ren, Z. J. Dai, C. J. Wu, and Y. H. Ji, “Xu J.IL-6,, IL-8 and TNF-alpha levels correlate with disease stage in breast cancer patients. Adv," Clinical and Experimental Medicine, vol. 26, no. 3, pp. 421-426, 2017.

[24] F. Wang, C. Tang, X. Gao, and J. Xu, "Identification of a sixgene signature associated with tumor mutation burden for predicting prognosis in patients with invasive breast 
carcinoma," Annals of Translational Medicine, vol. 8, no. 7, p. $453,2020$.

[25] J. Su, L. F. Miao, X. H. Ye, M. S. Cui, and X. F. He, “Development of prognostic signature and nomogram for patients with breast cancer," Medicine, vol. 98, no. 11, article e14617, 2019.

[26] H. Xu, R. Zou, F. Li et al., "MRPL15 is a novel prognostic biomarker and therapeutic target for epithelial ovarian cancer," Cancer Medicine, vol. 10, no. 11, pp. 3655-3673, 2021.

[27] X. Qiu, D. Guo, D. Juan, Y. Bai, and F. Wang, "A novel biomarker, MRPS12 functions as a potential oncogene in ovarian cancer and is a promising prognostic candidate," Medicine, vol. 100, no. 8, 2021.

[28] L. Zhuang, Z. Meng, and Z. Yang, "MRPL27 contributes to unfavorable overall survival and disease-free survival from cholangiocarcinoma patients," International Journal of Medical Sciences, vol. 18, no. 4, pp. 936-943, 2021.

[29] W. Jiang, C. Zhang, Y. Kang et al., "MRPL42 is activated by YY1 to promote lung adenocarcinoma progression," Journal of Cancer, vol. 12, no. 8, pp. 2403-2411, 2021.

[30] C. Hao, H. Duan, H. Li et al., "Knockdown of MRPL42 suppresses glioma cell proliferation by inducing cell cycle arrest and apoptosis," Bioscience Reports, vol. 38, no. 2, article BSR20171456, 2018.

[31] Z. Tao, H. Suo, L. Zhang et al., "Bo Chen.MRPL13 is a prognostic cancer biomarker and correlates with immune infiltrates in breast cancer," Oncotargets and Therapy, vol. 13, pp. 12255-12268, 2020.

[32] M. Cai, H. Li, R. Chen, and X. Zhou, "MRPL13 promotes tumor cell proliferation, migration and EMT process in breast cancer through the PI3K-AKT-mTOR pathway," Cancer Management and Research, vol. Volume 13, pp. 2009-2024, 2021.

[33] Y.-K. Lee, J. J. Lim, U.-w. Jeoun et al., "So Mee Kwon, Changhan Lee, Gyesoon YoonLactate-mediated mitoribosomal defects impair mitochondrial oxidative phosphorylation and promote hepatoma cell invasiveness," Journal of Biological Chemistry, vol. 292, no. 49, pp. 20208-20217, 2017.

[34] X. He, A. Zhou, H. Lu et al., "Suppression of mitochondrial complex I influences cell metastatic properties," PLoS One, vol. 8, no. 4, article e61677, 2013.

[35] D. Chandra and K. K. Singh, "Genetic insights into OXPHOS defect and its role in cancer," Biochimica et Biophysica Acta (BBA)-Bioenergetics, vol. 1807, no. 6, pp. 620-625, 2011.

[36] F. Schütz, S. Stefanovic, L. Mayer, A. von Au, C. Domschke, and C. Sohn, "PD-1/PD-L1 pathway in breast cancer," Oncology Research and Treatment, vol. 40, no. 5, pp. 294-297, 2017.

[37] A. Cimino-Mathews, J. B. Foote, and L. A. Emens, "Immune targeting in breast cancer," Oncology, vol. 29, no. 5, pp. 375385, 2015.

[38] L. Keren, M. Bosse, D. Marquez et al., “A structured tumorimmune microenvironment in triple negative breast cancer revealed by multiplexed ion beam imaging," Cell, vol. 174, no. 6, pp. 1373-1387.e19, 2018.

[39] S. Burugu, K. Asleh-Aburaya, and T. O. Nielsen, "Immune infiltrates in the breast cancer microenvironment: detection, characterization and clinical implication," Breast Cancer, vol. 24, no. 1, pp. 3-15, 2017.

[40] R. Kleef, A. Ralph Moss, M. Szasz, A. Bohdjalian, H. Bojar, and T. Bakacs, "Complete clinical remission of stage IV triplenegative breast cancer lung metastasis administering lowdose immune checkpoint blockade in combination with hyperthermia and interleukin-2," Integrative Cancer Therapies, vol. 17, no. 4, pp. 1297-1303, 2018.

[41] M. A. Postow, M. K. Callahan, and J. D. Wolchok, "Immune checkpoint blockade in cancer therapy," Journal of Clinical Oncology, vol. 33, no. 17, pp. 1974-1982, 2015.

[42] T. Bakacs and J. N. Mehrishi, "Anti-CTLA-4 therapy may have mechanisms similar to those occurring in inherited human CTLA4 haploinsufficiency," Immunobiology, vol. 220, no. 5, pp. 624-625, 2015.

[43] T. A. O'Meara and S. M. Tolaney, “Tumor mutational burden as a predictor of immunotherapy response in breast cancer," Oncotarget, vol. 12, no. 5, pp. 394-400, 2021.

[44] P. Schmid, S. Adams, H. S. Rugo et al., "and IMpassion 130 Trial Investigators. Atezolizumab and nab-paclitaxel in advanced triple-negative breast cancer," The New England Journal of Medicine, vol. 379, no. 22, pp. 2108-2121, 2018. 